\title{
Iron regulation of triosephosphate isomerase transcript stability in the yeast Saccharomyces cerevisiae
}

\author{
Klaus Krieger and Joachim F. Ernst
}

Author for correspondence: Joachim F. Ernst. Tel: +49 211311 5176. Fax: +49211311 5176/5370.

Institut für Mikrobiologie, Heinrich-Heine Universität, Universitätsstr. 1, Geb. 26.12, D-40225 Düsseldorf, FRG

\begin{abstract}
By differential hybridization we have identified CDNA clones that are derived from iron-regulated genes in Saccharomyces cerevisiae. Sequencing of seven CDNA clones revealed that five clones correspond to TPI1 encoding triosephosphate isomerase (Tpi1p) and one corresponds to TDH3 encoding glyceraldehyde-3-phosphate dehydrogenase (Tdh3p). During iron-limited growth mRNA levels for Tpi1p and Tdh3p were at least 3-fold lower than during iron-saturated growth; as shown with a hem1 mutant strain this regulation does not require haem synthesis. mRNA half-lives of TPI1 (TDH3) were $11.5 \mathrm{~min}$ (18 $\mathrm{min}$ ) in low-iron medium and $30 \mathrm{~min}$ ( $32.5 \mathrm{~min}$ ) in iigh-iron medium, indicating iron-regulation of transcript half-lives; the stabilities of the $A C T 1$ and PDC1 transcripts were not influenced by iron. Increased glycerol production during growth in low-iron, as compared to high-iron medium, is consistent with a modification of the glycolytic flux during iron-limited growth in S. cerevisiae.
\end{abstract}

Keywords: Iron, regulation, triosephosphate isomerase, transcript stability, Saccharomyces cerevisiae

\section{INTRODUCTION}

Iron is an essential element for most living cells. Redox reactions, such as reactions of the respiratory chain, as well as key reactions in lipid and nucleotide synthesis are critically dependent on iron. To make external iron available, most micro-organisms secrete specific ironchelators (siderophores) into the growth medium; the siderophore-iron chelates are efficiently transported into the microbial cells by specific uptake systems (for review see Winkelmann et al., 1987). Intracellularly, excess iron is stored by specific proteins, such as ferritin. Siderophores are not produced by the yeast Saccharomyces cerevisiae; its iron uptake system consists of a $\mathrm{Fe}^{3+}$ reductase and a $\mathrm{Fe}^{2+}$ membrane transporter (Lesuisse \& Labbe, 1989; Dancis et al., 1992; Eide \& Guarente, 1992).

Since iron is essential, but also toxic at high concentrations, it is not surprising that iron metabolism is strongly regulated. In bacteria, the fur protein represses transcription initiation at genes encoding proteins involved in iron uptake, if cells are grown in high-iron media (Braun et al., 1991). In mammalian cells, iron regulates its own

Abbreviations: DHAP, dihydroxyacetone phosphate; GAP, glyceraldehyde 3-phosphate. uptake by lowering the mRNA stability of the receptor for the iron-carrier transferrin, while stimulating translation of the storage protein ferritin (Owen \& Kühn, 1987; Hentze, 1991). Haem-dependence of translation in reticulocytes has long been recognized (Safer, 1983). In several fungal species the synthesis and the uptake of siderophores are stimulated under iron-limited conditions (Winkelmann et al., 1987). For S. cerevisiae, it has been reported that the $\mathrm{Fe}^{2+}$ transporter, but not the $\mathrm{Fe}^{3+}$ reductase is stimulated during iron-starvation (Eide \& Guarente, 1992), at variance with earlier reports (Dancis $e t$ al., 1992). Haem acts a co-effector to stimulate or to repress transcription of several S. cerevisiae genes (Zitomer \& Lowry, 1992).

While in previous reports iron has been found to regulate expression only of genes that are directly involved in iron uptake and its metabolism, we describe here iron regulation of glycolytic enzymes that are not known to directly participate in iron metabolism. The functional significance of this regulation may be to avoid NADH accumulation in iron-starved cells, whose respiratory chain functions inefficiently. Unlike the destabilization of the transferrin receptor mRNA by iron in mammalian cells (Owen \& Kühn, 1992), iron affects TPI1 expression in S. cerevisiae positively by stabilization of the TPI1 transcript. 


\section{METHODS}

Strains and media. The S. cerevisiae strains BJ1991 (MATa ura3-52 leu2 trp1 pep4-3 prb1-1122) (Jones, 1991), RY262 (MATa ura3-52 bis4-539 rpb1-1) (Nonet et al., 1987) and M10-1A (MAT $\alpha$ bem 1 trp5-48 met) (Matner \& Sherman, 1982) were used. Unless stated otherwise cells were grown in complex YPD or SD minimal media; amino acids were added to SI) media for auxotrophic strains (Sherman et al., 1986). Iron-free SD medium was prepared from concentrated stock solutions containing $10 \times$ nitrogen source/salts, $100 \times$ vitamins and $100 \times$ trace elements without $\mathrm{Fe}$. The nitrogen source/salts stock solution was extracted four times with $3 \%$ hydroxyquinoline in $\mathrm{CHCl}_{3}$, followed by several extractions with $\mathrm{CHCl}_{3}$ and autoclaving (Nicholas, 1957). Glass vessels to contain deferrated media were prepared by washing with $5 \mathrm{M}$ $\mathrm{HCl}$ and rinsing with glass-distilled water; polypropylene vessels were pretreated by rinsing with $20 \mathrm{mM}$ EDTA and distilled water. Iron was added in the form of a freshly filtered solution of $\mathrm{FeSO}_{4}$ in water $(13.2 \mu \mathrm{M}$ for iron-saturated and $0.13 \mu \mathrm{M}$ for iron-limited growth). To permit haem biosynthesis in strain M10-1A it was supplemented with $\delta$-aminolevulinic acid $\left(100 \mu \mathrm{l}\right.$ of a $5 \mathrm{mg} \mathrm{ml}^{-1}$ stock solution per $\left.100 \mathrm{ml}\right)$; growth without haem biosynthesis was in media supplemented with ergosterol and Tween $80(50 \mu$ lof a stock solution containing $144 \mathrm{mg}$ ergosterol and $14 \mathrm{ml}$ Tween 80 in $36 \mathrm{ml}$ ethanol were added to $1 \mathrm{ml}$ of medium).

CDNA and screening procedures. Strain BJ1991 was pregrown in deferrated SD medium to deplete internal iron stores. This culture was used to inoculate deferrated $\mathrm{SD}$ medium, or deferrated SD medium containing $10 \mu \mathrm{g} \mathrm{FeSO}_{4} \mathrm{ml}^{-1}(36 \mu \mathrm{M})$. Cells were harvested in the exponential growth phase $\left(\mathrm{OD}_{600} \approx 0.5\right)$ and total RNA was prepared as described by Trumpower et al. (1990). The mRNA fraction was isolated using a commercial mRNA purification kit (Pharmacia). Standard procedures were used to prepare a cDNA bank and for differential hybridization (Sambrook et al., 1989). Briefly, cDNA was synthesized using the poly(A) RNA of iron-saturated cells as template and EcoRI adapters were added using a cDNA synthesis kit (Pharmacia). The tailed cDNA was ligated to the arms of EcoRI-cut lambda gt10 (Genofit), packaged in vitro (using Packagene, Promega) and transfected into E. coli C600 $b f t$. Phages were plated and transferred to duplicate nitrocellulose filters as described in Sambrook et al. (1989). Radioactive probes were prepared by including $\left[{ }^{32} \mathrm{P}\right] \mathrm{ATP}$ in the firststrand cDNA synthesis reaction with the poly(A) RNAs as templates. One replica filter was hybridized with radioactive cDNA of iron-starved cells, the other with cDNA of ironsaturated cells. Phages hybridizing strongly with the $+\mathrm{Fe}$ cDNA, but weakly with the cDNA of Fe-starved cells were picked and rescreened. DNA of identified phages was prepared and analysed according to standard protocols (Sambrook $e t$ al., 1989).

RNA procedures. Strain RY262 containing a thermosensitive RNA polymerase II (Nonet et al., 1987) was used to determine transcript stabilities, following the protocol described by Parker et al. (1991). RY262 was pregrown in iron-depleted medium; this culture was used to inoculate $300 \mathrm{ml}$ of iron-depleted SD medium supplemented to contain either $0.13 \mu \mathrm{M}$ or $13.2 \mu \mathrm{M}$ $\mathrm{FeSO}_{4}$. The cultures were grown at $26^{\circ} \mathrm{C}$ to a density of $10^{7}$ cells $\mathrm{ml}^{-1}$. Portions $(50 \mathrm{ml}$ ) of the cultures were then shifted to $36{ }^{\circ} \mathrm{C}$ and incubated for different times. Cells were harvested by centrifugation at 10000 r.p.m. for $30 \mathrm{~s}$ in a precooled JA-20 rotor (Beckman), immediately frozen using liquid nitrogen and stored at $-20^{\circ} \mathrm{C}$ before preparation of RNA. RNA was isolated from the frozen cells using the method of Trumpower ef al. (1990) and Northern blots were prepared according to stanctard procedures (Sambrook et al., 1989) using labelled DNA probes. As probes, the $563 \mathrm{bp}$ ClaI fragment containing ACT1 coding sequences (Gallwitz \& Sures, 1980 ), or a $0.7 \mathrm{~kb}$ EcoRI fragment of pPDC1 (kindly supplied by J. Heinisch, Institut für Mikrobiologie, Univ. Düsseldorf) containing PDC1 sequences, or cDNA inserts carrying TPI1, TPH3 or LEU2 sequences were used. Ribosomal RNA was detected using labelled DNA of phage lambda gp21-Sc310 (Philippsen et al., 1978) containing rDNA. RNA hybridization signals were quantitated using a Molecular Dynamics laser densitometer. The ratio of specific transcript/rRNA was calculated for each sample and the ratios at time points $\mathrm{t}=0 \mathrm{~min}$ after the temperature shift were arbitrarily assigned the value $100 \%$ (relative mRNA level).

Glycerol assay. Glycerol secreted by yeast strains into the growth medium was determined using a commercial kit (Boehringer) and expressed as ng glycerol per cell.

\section{RESULTS}

\section{Screening for iron-regulated genes}

In preparing cDNA bank constructions we established conditions for iron-limiting and iron-saturated growth of $S$. cerevisiae. Due to internal iron stores the growth of $S$. cerevisiae initially was not reduced in iron-depleted medium, if an inoculum of iron-saturated cells was used. However, if strain BJ1991 was pregrown extensively in deferrated medium, its growth became iron-dependent during subsequent culturing. Maximal growth was obtained at iron concentrations $>0.89 \mu \mathrm{M}$; at lower iron concentrations growth was reduced, due to iron starvation (Eide \& Guarente, 1992).

Differential hybridization was used to identify $S$. cerevisiae genes whose expression is changed as a consequence of alterations in environmental iron levels. mRNA of cells grown in medium containing high iron levels $(36 \mu \mathrm{M})$ was isolated and a cDNA bank in bacteriophage lambda gt 10 was constructed. Filter duplicates carrying recombinant phages were hybridized with radioactive $\mathrm{cDNAs}$ derived from iron-starved or iron-saturated cells. Among 16000 phages, seven were identified that repeatedly showed strong signals with cDNA of iron-grown cells, while reacting weakly with cDNA of iron-starved cells (lambda 2, 7, 9, 15, 23, 34, 35) (Fig. 1).

DNA of the seven phages identified was prepared, digested with EcoRI (which cuts the vector once at the cDNA insertion point) and the cDNA insert analysed by agarose gel electrophoresis (data not shown). Lambda 2, 7, 9, 15 and 23 contained a single EcoRI cDNA insert of about $1.2 \mathrm{~kb}$, while lambda 34 contained two fragments of about 1.2 and $1.0 \mathrm{~kb}$ and lambda 35 contained three fragments of about $1 \cdot 7,1.5$ and $1.2 \mathrm{~kb}$. To explore if any of the recombinant phages was identical, insert DNA of each individual bacteriophage was radioactively labelled and hybridized against DNA of the other phages in a dotblot experiment. This analysis revealed that phages lambda 2, 7, 15, 23 and 35 contained identical cDNA sequences, while lambda 9 and 34 were different.

The EcoRI inserts of bacteriophages lambda 2 and 34 were inserted into M13mp8 and sequenced. This analysis revealed a $100 \%$ match of the lambda 2 insert to the $S$. 


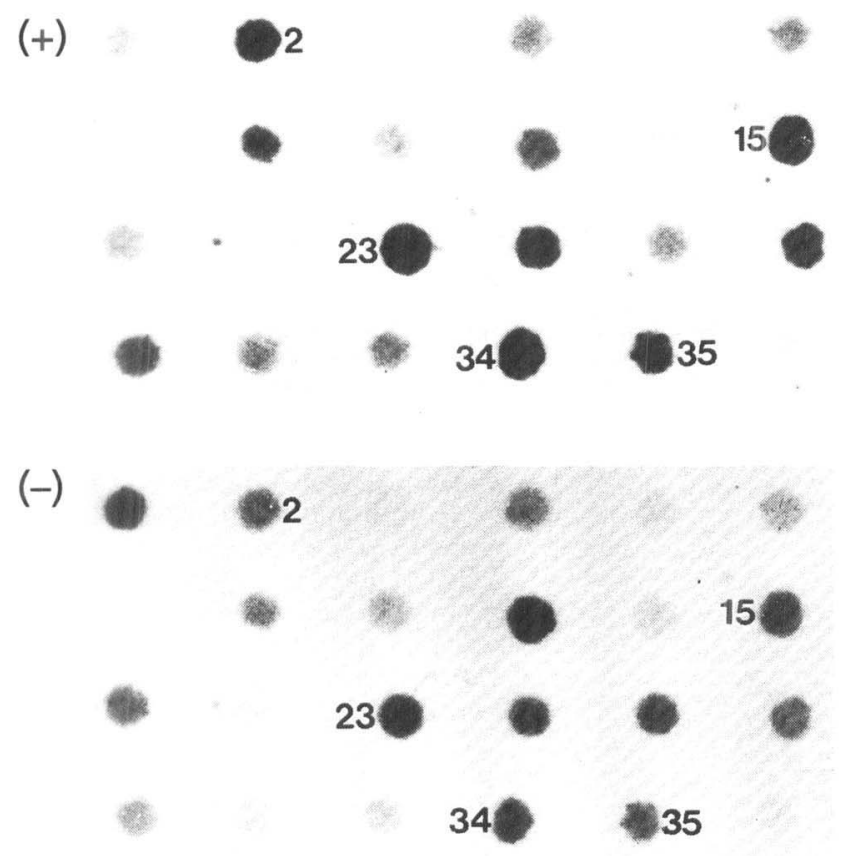

Fig. 1. Identification of recombinant bacteriophages containing CDNA of iron-regulated $S$. cerevisiae genes. DNA of bacteriophage lambda gt10 derivatives, identified in a primary screen, was spotted on duplicate nylon membranes and hybridized with radioactive CDNA derived from iron-saturated $(+)$ or iron-limited $(-)$ cells. Numbers indicate bacteriophage DNAs with greater hybridization to $(+) \mathrm{Fe}$ CDNA as compared to (-)Fe CDNA.

cerevisiae TPI1 gene encoding triosephosphate isomerase (Alber \& Kawasaki, 1982); for lambda 34 a 100\% match to the $S$. cerevisiae TDH3 encoding glyceraldehyde-3phosphate (GAP) dehydrogenase (pgap49) was found (Holland \& Holland, 1979; McAlister \& Holland, 1985). Thus, two genes encoding glycolytic proteins were identified whose transcript levels are regulated positively by iron in the growth medium. Since $T D H 3$ is a member of three closely homologous genes in $S$. cerevisiae (McAlister \& Holland, 1985) we concentrated on regulation of the unique TPI1 gene in the following RNA analyses.

\section{Effects of iron and haem on TPI1 transcript levels}

Haem has been described as a potent regulatory molecule for transcription in S. cerevisiae (Zitomer \& Lowry, 1992). It appeared possible that the observed regulation of the TPI1 and TDH 3 transcripts was due to low levels of haem in iron-starved cells and high levels in iron-saturated cells. To test this possibility we tested iron regulation in a bem 1 mutant of $S$. cerevisiae (M10-1A) that is defective in $\delta$-aminolevulinate synthase. This strain is able to grow without significant haem synthesis on glucose media containing ergosterol and a source of unsaturated fatty acids (Tween 80); the expression of haem-dependent genes, e.g. CYC1, is reduced at least 200 -fold under these growth conditions (Guarente \& Mason, 1983). It can be (a)

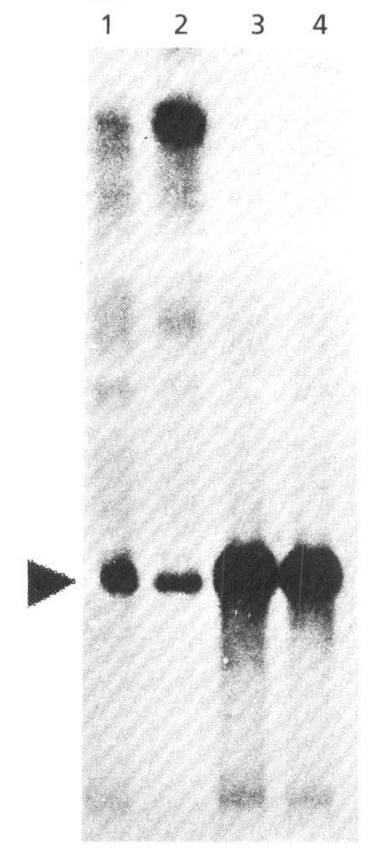

(b)

$\begin{array}{llll}1 & 2 & 3 & 4\end{array}$

Fig. 2. Effects of iron and haem on transcript levels. Transcript levels of the LEU2 (a) and TPI1 (b) genes were determined. RNA was isolated and analysed from strain M10-1A grown without haem biosynthesis in high-iron medium (lane 1) or low-iron medium (lane 2). In addition, RNA from iron-saturated cells (grown in YPD) not synthesizing haem (lane 3) or synthesizing haem (lane 4) was isolated and analysed. Northern blots were probed using LEU2 (a) or TPI1 (b) as probes. The respective transcripts are indicated by arrows.

seen in Fig. 2 that the level of the LEU2 gene transcript as a control is not altered by iron in the growth medium (Fig. 2a, lanes 1,2). On the other hand, high levels of the TPI1 transcript depend on iron sufficiency, even if no haem biosynthesis occurs (Fig. 2b, lanes 1,2); similarly, the TDH3 transcript (possibly including transcripts of the $T D H 1$ and $T D H 2$ genes) is iron-dependent (data not shown). From separate experiments we estimate the TPI1 transcript level is altered by environmental iron by a factor of 3 to 7. No differences in LEU2, TPI1 and TDH3 transcript levels were observed if iron-saturated cells were able (addition of $\delta$-aminolevulinate) or were unable to synthesize haem (Fig. 2, lanes 3,4). Thus, these results demonstrate that the TPI1 and TDH 3 transcript levels are related to iron levels in the environment, but are independent of haem biosynthesis.

\section{Iron regulation of TPI1 transcript stability}

Conceivably, the different levels of the TPI1 and TDH3 transcripts during iron-limited and iron-saturated growth are due to differences in mRNA synthesis and/or mRNA stability. We tested the latter possibility using strain RY262, which contains the rbp1 mutation encoding a thermosensitive RNA polymerase II (Nonet et al., 1987). Cells pregrown at $26^{\circ} \mathrm{C}$ in deferrated medium were used to inoculate medium containing $0.13 \mu \mathrm{M}$ or $13.2 \mu \mathrm{M}$ iron. 


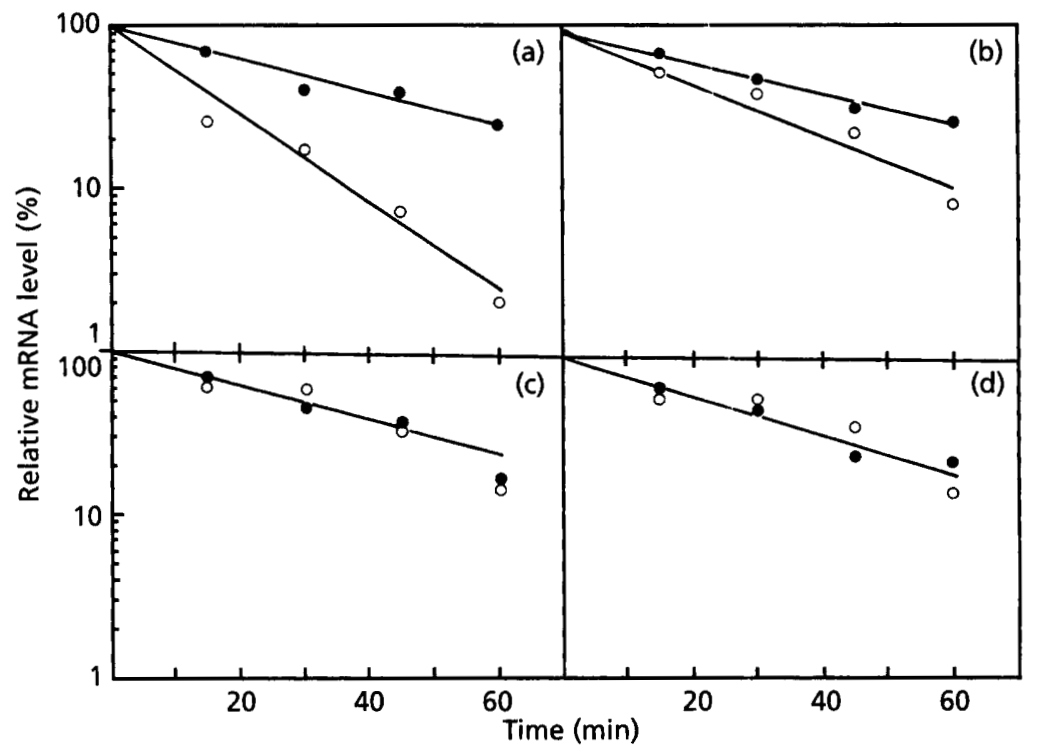

Fig. 3. mRNA half-lives in iron-starved and iron-saturated cells. Strain RY262 was grown in medium containing $0.13 \mu \mathrm{M}$ or $13.2 \mu \mathrm{M}$ $\mathrm{FeSO}_{4}$ at the permissive temperature $\left(26^{\circ} \mathrm{C}\right)$. At time $t=0 \mathrm{~min}$, the cells were shifted to $36^{\circ} \mathrm{C}$ and RNA was isolated after $0,15,30$, 45 and 60 min incubation. RNA was analysed by Northern blotting using specific probes and autoradiographic signals were quantified using laser densitometry. The mRNAVrRNA ratio at $t=0$ min was arbitrarily assigned the value $100 \%$. (a) TPI1; (b) TDH3; (c) $A C T 1$; (d) PDC1; 0 , iron-saturated cells; $\bigcirc$, iron-starved cells.
Table 1. Transcript half-lives in different media

Transcript half-lives were calculated from Fig. 3.

\begin{tabular}{|clc|}
\hline Gene & $\begin{array}{c}\text { Transcript half-life } \\
\text { (min) }\end{array}$ \\
\cline { 2 - 3 } & $\begin{array}{c}\mathbf{0 . 1 3} \boldsymbol{\mu M} \\
\mathbf{F e S O}_{4}\end{array}$ & $\begin{array}{c}\mathbf{1 3 \cdot 2} \boldsymbol{\mu \mathbf { M }} \\
\mathbf{F e S O}_{\mathbf{4}}\end{array}$ \\
\hline TPI1 & $11 \cdot 5$ & 30 \\
$T D H 3$ & 18 & $32 \cdot 5$ \\
$A C T 1$ & 32 & 32 \\
PDC1 & 26.5 & 26.5 \\
\hline
\end{tabular}

After growth at $26^{\circ} \mathrm{C}$ to the exponential growth phase the cultures were shifted to the non-permissive temperature $\left(36{ }^{\circ} \mathrm{C}\right)$. At different times of incubation mRNA was isolated, and was then analysed in 'Northern' experiments using specific probes. To correct for differences in mRNA concentrations the resulting autoradiographs were evaluated using laser densitometry and, for each transcript, ratios of the specific hybridization signal to rRNA were determined. The ratio at the time $=0 \mathrm{~min}$ at the nonpermissive temperature was arbitrarily assigned the value 100.

It can be seen in Fig. 3(c) that following transcriptional arrest the level of the ACT1 transcript declined exponentially. mRNA half-life was $32 \mathrm{~min}$, irrespective of whether cells were grown in high or low iron concentrations. Likewise, the transcript half-life of the glycolytic gene PDC1 encoding pyruvate decarboxylase was not influenced by the iron status of the cells (half-life about $27 \mathrm{~min}$ for both conditions) (Fig. 3d). In contrast, clear differences in mRNA half-lives could be determined for TPI1 (Fig. 3a). Under iron-limited growth the mRNA half-life was $11 \mathrm{~min}$, while during iron-saturated growth, it increased to $30 \mathrm{~min}$. An increase in mRNA stability in high-iron medium, although not as extreme, was detected for the TDH3 transcript (Fig. 3b). For TDH3, transcript half-life was $33 \mathrm{~min}$ in high-iron medium and about $18 \mathrm{~min}$ in low-iron medium. However, for TDH3 crosshybridizations with the related genes TDH1 and TDH2 cannot be excluded. The results, summarized in Table 1 , demonstrate that the stability of the TPI1 and, possibly, the TDH3 transcript, is positively regulated by the presence of iron in the growth medium. In a separate experiment the observed 3-fold/2-fold increases of TPI1/TDH3 half-lives was repeated (data not shown). Iron regulation does not extend to other glycolytic and non-glycolytic genes, as shown for PDC1 and ACT1 (Fig. 3c, d).

\section{Iron regulation of glycerol production}

A physiological consequence of lowered TPI1 and TDH3 expression in iron-starved cells could be an altered glycolytic flux compared to iron-saturated cells. Lack of triosephosphate isomerase could lead to higher dihydroxyacetone phosphate (DHAP) levels, especially if a reduced level of GAP dehydrogenase lowered the catabolism of GAP. Thus, both defects could act in a similar direction by directing the glycolytic flux to DHAP and, ultimately, to glycerol. This hypothesis was tested by the determination of glycerol levels in the medium of ironsaturated or iron-starved cells of strain BJ1991 (Table 2). The data show that glycerol levels produced by cells are a factor of 5 to 6 lower in iron-saturated cells as compared to iron-starved cells in deferrated medium containing no added iron. Cells growing in a medium containing $0 \cdot 18 \mu \mathrm{M} \mathrm{FeSO}_{4}$ produce twice the amount of glycerol as compared to growth in a medium containing $0.89 \mu \mathrm{M}$ $\mathrm{FeSO}_{4}$. In separate experiments with strain BJ1991, as well as strains RY262 and M10-1A, enhanced glycerol production at low levels of iron in the medium could be reproduced (data not shown). However, absolute 
Table 2. Glycerol production in media containing different iron concentrations

Strain BJ1991, pregrown in deferrated medium, was inoculated in media containing different iron concentrations. After $69 \mathrm{~h}$ incubation, the cell density and the amount of glycerol in the medium was determined.

\begin{tabular}{|ccc|}
\hline $\begin{array}{c}\mathrm{FeSO}_{4} \text { in } \\
\text { medium } \\
(\boldsymbol{\mu} \mathbf{M})\end{array}$ & $\begin{array}{c}\text { Cell density } \\
\left(\text { cells ml } \mathbf{~}^{-1}\right)\end{array}$ & $\begin{array}{c}\text { Glycerol } \\
\text { production } \\
(\text { ng per cell })\end{array}$ \\
\hline 0 & $4 \times 10^{5}$ & 250 \\
$0 \cdot 18$ & $7 \times 10^{6}$ & 38 \\
$0 \cdot 89$ & $7 \times 10^{6}$ & 18 \\
\hline
\end{tabular}

amounts of glycerol produced varied several-fold between experiments, presumably because minor differences in culturing conditions can have a significant effect on intracellular iron levels and thereby, on glycerol production.

\section{DISCUSSION}

We describe here that TPI1 and TDH3 transcripts are stabilized in the presence of iron and destabilized during iron deprivation. Although transcriptional regulation cannot be excluded it is possible that the lowered TPI1 transcript stability in low-iron medium is sufficient to explain the lower steady-state transcript levels compared to iron-saturated growth. In agreement with this notion, TPI1 and TDH2 transcript levels are not altered significantly by different growth rates (Moore et al., 1991). Besides the iron-controlled transcript stabilities described here, it has been reported that the transcript for succinate dehydrogenase in S. cerevisiae is stabilized in the presence of glucose (Lombardo et al., 1992). In mammalian cells, iron regulates the stability of the transferrin receptor mRNA (Owen \& Kühn, 1987); however, in this case iron acts in the opposite sense, by transcript destabilization. The molecular mechanisms of the observed iron regulation of TPI1 transcript stability remain to be determined. By using a bem 1 mutant we have shown that the change in TPI1 transcript levels caused by iron does not require haem synthesis. Conceptually, iron could activate a cellular component required for transcript stability; alternatively, the absence of iron during iron-limitation could activate a mRNA-degrading activity. More indirect mechanisms for mRNA destabilization are also possible, such as reduced translation of TPI1 during iron-limited growth, as has been described for mammalian cells (Daar \& Maquat, 1988).

Iron limitation is a growth condition frequently encountered by micro-organisms in natural environments. This occurs because iron forms insoluble polymeric hydroxides at or around neutral $\mathrm{pH}$ values. The yeast $S$. cerevisiae is particularly affected by iron limitation since it does not produce siderophores that may solubilize $\mathrm{Fe}^{3+}$; however, heterologous siderophores secreted by other micro-organisms, such as ferrioxamine B, may be used by S. cerevisiae (Lesuisse et al., 1987). One of the immediate consequences of iron deprivation will be defective function of the respiratory chain, which consists of a series of haem and non-haem iron proteins. S. cerevisiae may cope with the resulting accumulation of NADH and a lack of $\mathrm{NAD}^{+}$regeneration under such growth conditions by lowering the rate of NADH production and by providing additional electron acceptors. The reduced expression of both TPI1 and TDH3 during iron-limited growth that we have observed serves this physiological goal. Reduced TPI1 expression will lower the conversion of DHAP into GAP while reduced $T D H 3$ expression will lower further processing of GAP. The accumulated DHAP may serve as an acceptor for excessive NADH electrons, leading to elevated glycerol production during iron-limited growth, as we have shown. The reduced glycolytic flux during iron starvation may be complemented by lowered activity of aconitase (an iron-dependent enzyme) (Constable $e t$ al., 1992) leading to a slow-down of endoxidation in the tricarboxylic acid cycle, again lowering NADH production. The combined effects of the described alterations in enzyme levels, or enzyme activities, may allow continued respiration and growth of $S$. cerevisiae in conditions of iron limitation.

Although the above theory is consistent with the data obtained so far, the levels of the TPI1 and TDH3 proteins, as well as the enzymic activities of both enzymes in different states of iron availability remain to be determined. One difficulty of such studies will be the clear definition of the physiological iron status of the yeast cell; this is difficult because of significant internal iron stores and the continuous change in the intracellular iron status during growth. It also remains to be determined whether $T D H 1$ and $T D H 2$ transcripts are regulated by iron as their homologue $T D H 3$. Finally, it will be of interest to determine the transcript target sites, as well as the cellular components that are involved in iron regulation of transcript stability in $S$. cerevisiae.

\section{REFERENCES}

Alber, T. \& Kawasaki, G. (1982). Nucleotide sequence of the triose phosphate isomerase gene of Saccharomyces cerevisiae. $\int \mathrm{Mol}$ Appl Genet 1, 419-434.

Braun, V., Günter, K. \& Hantke, K. (1991). Transport of iron across the outer membrane. Biol Metl 4, 14-22.

Constable, A., Quick, S., Gray, N. K. \& Hentze, M. W. (1992). Modulation of the RNA-binding activity of a regulatory protein by iron in vitro: switching between enzymatic and genetic function? Proc Natl Acad Sci USA 89, 4554-4558.

Daar, I. O. \& Maquat, L. E. (1988). Premature translation termination mediates triosephosphate isomerase mRNA degradation. Mol Cell Biol 8, 802-813.

Dancis, A., Roman, D. G., Anderson, G. J., Hinnebusch, A. G. \& Klausner, R. D. (1992). Ferric reductase of Saccharomyces cerevisiae: molecular characterization, role in iron uptake, and transcriptional control by iron. Proc Natl Acad Sci US A 89, 3869-3873.

Eide, D. \& Guarente, L. (1992). Increased dosage of a transcriptional activator gene enhances iron-limited growth of Saccharomyces cerevisiae. J Gen Microbiol 138, 347-354. 
Gallwitz, D. \& Sures, I. (1980). Structure of a split yeast gene: complete nucleotide sequence of the actin gene in Saccharomyces cerevisiae. Proc Natl Acad Sci US A 77, 2546-2550.

Guarente, L. \& Mason, T. (1983). Heme regulates transcription of the CYC1 gene of $S$. cerevisiae via an upstream activation site. Cell 32, 1279-1286.

Hentze, M. (1991). Determinants and regulation of cytoplasmic mRNA stability in eukaryotic cells. Biocbim Biophys Acta 1090, 281-292.

Holland, J. P. \& Holland, M. J. (1979). The primary structure of a glyceraldehyde-3-phosphate dehydrogenase gene from Saccharomyces cerevisiae. J Biol Chem 254, 9839-9845.

Jones, E. W. (1991). Tackling the protease problem in Saccbaromyces cerevisiae. Methods Enzymol 194, 428-453.

Lesuisse, E. \& Labbe, P. (1989). Reductive and non-reductive mechanisms of iron assimilation by the yeast Saccharomyces cerevisiae. J Gen Microbiol 135, 257-263.

Lesuisse, E., Raguzzi, F. \& Crichton, R. R. (1987). Iron uptake by the yeast Saccharomyces cerevisiae: involvement of a reduction step. $J$ Gen Microbiol 133, 3229-3236.

Lombardo, A., Cereghino, G. P. \& Scheffler, I. E. (1992). Control of mRNA turnover as a mechanism of glucose repression in Saccharomyces cerevisiae. Mol Cell Biol 12, 2941-2948.

McAlister, L. \& Holland, M. J. (1985). Isolation and characterization of yeast strains carrying mutations in the glyceraldehyde-3phosphate dehydrogenase genes. J Biol Chem 260, 15013-15018.

Matner, R. R. \& Sherman, F. (1982). Differential accumulation of two apo-iso-cytochromes $c$ in processing mutants of yeast. $J$ Biol Chem 257, 9811-9821.

Moore, P. A., Sagliocco, F. A., Wood, R. M. C. \& Brown, A. J. P. (1991). Yeast glycolytic mRNAs are differentially regulated. Mol Cell Biol 11, 5330-5337.

Nicholas, D. J. D. (1957). Microbiological methods for determining magnesium, iron, copper, zinc, manganese, and molybdenum. Methods Enzymol 3, 1035-1041.

Nonet, M., Scafe, C., Sexton, J. \& Young, R. (1987). Eucaryotic RNA polymerase conditional mutant that rapidly ceases mRNA synthesis. Mol Cell Biol 7, 1602-1611.

Owen, D. \& Kühn, L. C. (1987). Noncoding $3^{\prime}$ sequences of the transferrin receptor gene are required for mRNA regulation by iron. EMBO J 6, 1287-1293.

Parker, R., Herrick, D., Peltz, S. W. \& Jacobson, A. (1991). Measurement of mRNA decay rates in Saccharomyces cerevisiae. Methods Enzymol 194, 415-423.

Philippsen, P., Thomas, M., Kramer, R. A. \& Davis, R. W. (1978). Unique arrangement of coding sequences for $5 S, 5.8 S, 18 \mathrm{~S}$ and $25 \mathrm{~S}$ ribosomal RNA in Saccharomyces cerevisiae as determined by R-loop and hybridization analysis. $J$ Mol Biol 123, 387-404.

Safer, B. (1983). 2B or not 2B: regulation of the catalytic utilization of eIF-2. Cell 33, 7-8.

Sambrook, J., Fritsch, T. \& Maniatis, T. (1989). Molecular Cloning. A Laboratory Manual, 2nd edn. Cold Spring Harbor, NY: Cold Spring Harbor Laboratory.

Sherman, F., Fink, G. R. \& Hicks, J. B. (1986). Methods in Yeast Genetics. Cold Spring Harbor, NY: Cold Spring Harbor Laboratory.

Trumpower, B. L., Schmitt, M. E. \& Brown, T. A. (1990). A rapid and simple method for preparation of RNA from Saccharomyces cerevisiae. Nucleic Acids Res 18, 3091-3092.

Winkelmann, G., van der Helm, D. \& Neilands, J. B. (editors) (1987). Iron Transport in Microbes, Plants and Animals. New York: $\mathrm{VCH}$ Verlagsgesellschaft.

Zitomer, R. S. \& Lowry, C. V. (1992). Regulation of gene expression by oxygen in Saccharomyces cerevisiae. Microbiol Rev 56, 1-11.

Received 15 July 1993; revised 17 November 1993; accepted 24 November 1993. 